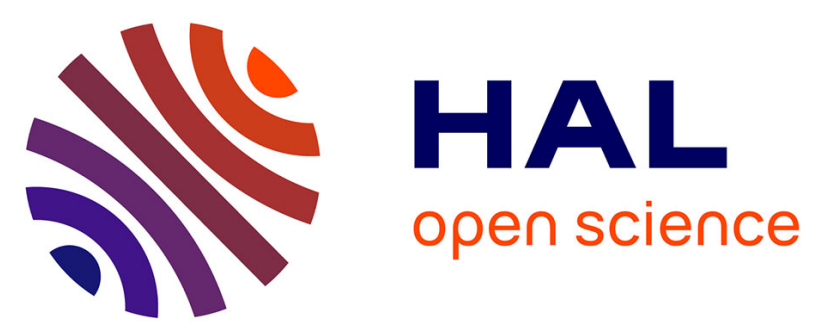

\title{
Improvement of toolpath quality combining polynomial interpolation with reduction of toolpath points
}

Hichem Bouchenitfa, Julien Chaves-Jacob, Jean-Marc Linares, Jean-Michel

Sprauel, Noureddine Azzam, Salim Boukebbab

\section{To cite this version:}

Hichem Bouchenitfa, Julien Chaves-Jacob, Jean-Marc Linares, Jean-Michel Sprauel, Noureddine Azzam, et al.. Improvement of toolpath quality combining polynomial interpolation with reduction of toolpath points. International Journal of Advanced Manufacturing Technology, 2015, 78, pp.875 883. 10.1007/s00170-014-6696-4 . hal-01463417

\section{HAL Id: hal-01463417 \\ https://hal-amu.archives-ouvertes.fr/hal-01463417}

Submitted on 9 Feb 2017

HAL is a multi-disciplinary open access archive for the deposit and dissemination of scientific research documents, whether they are published or not. The documents may come from teaching and research institutions in France or abroad, or from public or private research centers.
L'archive ouverte pluridisciplinaire HAL, est destinée au dépôt et à la diffusion de documents scientifiques de niveau recherche, publiés ou non, émanant des établissements d'enseignement et de recherche français ou étrangers, des laboratoires publics ou privés. 


\section{Improvement of toolpath quality combining polynomial interpolation with}

reduction of toolpath points

Hichem BOUCHENITFA ${ }^{a}$, Julien CHAVES-JACOB ${ }^{\mathrm{b} *}$, Jean-Marc LINARES ${ }^{\mathrm{b}}$, JeanMichel SPRAUEL ${ }^{b}$, Noureddine AZZAM ${ }^{b c}$, Salim BOUKEBBAB ${ }^{d}$

${ }^{a}$ Mouloud Mammeri University of Tizi-Ouzou, 15000, Tizi-Ouzou, Algeria

${ }^{b}$ Aix-Marseille Université, CNRS, ISM UMR 7287, 13288, Marseille cedex 09, France

${ }^{c}$ Department of Mechanical Engineering, Faculty of Sciences and Technology, University of Constantine 1, Ain-el-Bey Road, 25000 Constantine, Algeria.

${ }^{d}$ Department of Engineering Transport, Faculty of Sciences and Technology, University of Constantine 1, Ain-el-Bey road, 25000 Constantine, Algeria.

\section{${ }^{*}$ Corresponding author}

Abstract:

The aim of this study is to propose a 5-axis toolpath smoothing method in order to improve the quality of machined surfaces. Currently, toolpaths are commonly computed from CAD models presenting small geometrical discontinuities. These discontinuities may be caused by an insufficient quality of the CAD model (geometrical discontinuities) and the use of meshed surfaces (e.g. STL files). Normally, CAM systems generate linearly interpolated toolpaths. CNC options are then used on the machine to smooth the toolpath. The geometrical discontinuities of CAD models and linear toolpath interpolation may induce a unsmooth toolpath. This type of toolpath causes marks on the machined workpiece even if classical enhanced CNC options are used. Generally, these marks are unacceptable for the functionality of the workpiece. To reduce this problem, this study proposes a method to efficiently smooth toolpaths and consequently improve the obtained surface quality. The proposed method may be employed with high end controllers commonly used on 5-axis CNC machines. First, a five degree polynomial interpolation method is presented. This interpolation is computed to ensure geometrical continuity in the slope and curvature of the obtained toolpath. Next, a concatenation method is proposed to reduce the size of the CNC program and to improve the toolpath smoothness. Moreover, the purpose of this concatenation is to obtain an optimized repartition of points along the toolpath. Furthermore, in a reverse engineering process this method avoids surface reconstruction, decreasing the process time and improving the quality of the obtained surface. The efficiency of these methods is validated by the machining of biomedical prostheses. The CAD model used for the test is a meshed surface. 
Keywords:

Toolpath; 5-axis milling; Polynomial interpolation; Meshed surface; Free-form surfaces; Biomedical.

\section{Introduction}

Currently, the CAD model is essential to the machining of a free-form surface.

Subsequently this model is exported to CAM software to permit toolpath computation. However, To complete this transfer, it is sometimes necessary to use exchange formats. In the case of STL data that builds meshed surfaces, a lot of geometrical discontinuities are then present in the resulting CAD model. Furthermore, meshed CAD models may also be obtained by 3D-acquisition in a reverse engineering process. Figure 1 presents the different solutions to machine a surface in a reverse engineering approach.

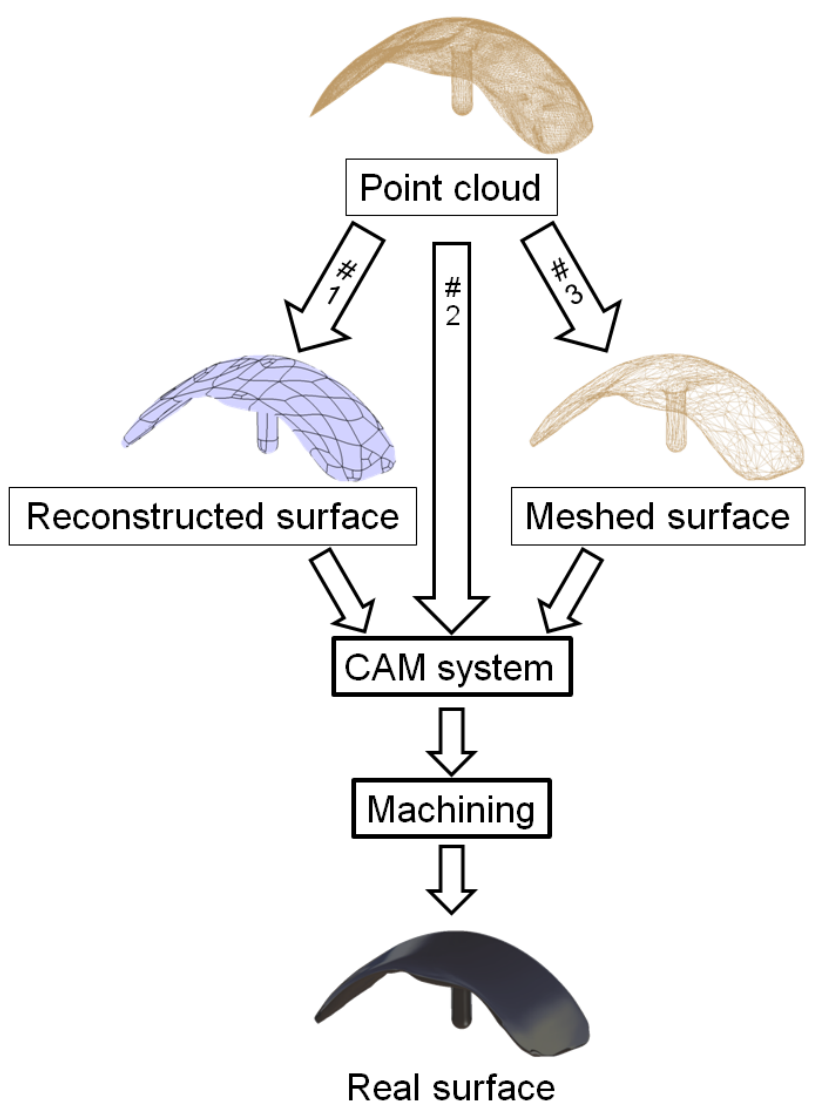

Fig.1 Possible solutions to machine a point cloud.

\#1: the historical solution starts with the surface reconstruction based on point clouds or meshed surfaces. Lim et al. [1] proposed a review of different techniques and soft computing methods for surface reconstruction. The author insisted on the importance of pre-processing 
steps such as compression and simplification. Furthermore, the author highlighted the errors introduced by the reconstruction step. After the reconstruction step, the toolpath is computed on the reconstructed surface. This solution imposes the surface reconstruction step, which is tedious and may introduce large errors between the measured points and the reconstructed surface. Generally, an approximation or an interpolation is used to reconstruct the surface.

\#2: the second solution is to directly compute the toolpath on the point cloud. Park et al. [2] proposed a method for this technique including on rough and finishing toolpaths. These methods are based on the slicing of the point cloud and the determination of machining regions applying an algorithm which calculates the boundary of a point cloud. On the other hand, the method proposed by Zhang et al. [3] consists in projecting an initial toolpath onto the point cloud. Then, an interpolation with Bezier curves is performed to smooth the obtained toolpath. Freg et al. [4] proposed a method to compute an iso-planar toolpath directly on a point cloud. This toolpath is obtained using a CL-net cell boundary, which is a pattern of rectangular cells. These patterns support the toolpath and are determined to ensure the specified machining error and scallop height constraints. Chiu et al. [5] used a method to construct a triangular meshed surface from point clouds. Subsequently, the computed toolpath is based on this meshed surface using toolpath smoothing with Biarc curves.

\#3: the third solution is based on a meshed surface (generally triangular) to compute the toolpath. The advantages of this technique, compared to the first, are time reduction and improvement of geometrical accuracy. Certainly, a point cloud is easier to best fit by a triangular pattern than a free-form surface (Bezier, Bspline, Nurbs...). Sun et al. [6] proposed a method to compute 5-axis iso-parametric toolpaths on triangular meshed surfaces. This method is based on the parameterization of the meshed surface via a harmonic map method. Park [7] presented a method to compute a toolpath on meshed sculptured surfaces. This method begins with the determination of the CL (Cutter Location) surface: place of driven points. Subsequently, a 2D toolpath is projected onto the meshed surface using a vertical slicing strategy. Jun et al. [8] also began computing the CL surface. This CL surface is meshed and then sliced by parallel planes to obtain the toolpath. Kim et al. [9] also defined a method based on the CL surface to machine meshed surfaces. This method deforms the CL surface to obtain a constant scallop height toolpath. Gouging problem is one of the main difficulties in the 5-axis toolpath computing on meshed surfaces. Lauwers et al. [10] proposed a method to compute toolpath directly on meshed model. The method starts computing the toolpath without considering gouging and after the tool position is adapted to suppress the gouging problem. The tool position adaptation is carried out in tool lift and orientation. Lu et al. [11] defined a first toolpath using the step-over distance to ensure the required machining tolerance. Thereafter, the tool position is modified to avoid gouging. 
Kiswanto et al. [12] proposed to lift the tool to avoid gouging. An algorithm was developed to determine the lift value of the tool based on the type of gouging.

Lasemi et al. [13] explained that currently the use of the second and third solutions is emerging (figure 1). Certainly, in reverse engineering, this method helps avoiding surface reconstruction before CAM process. Accordingly, in these methods, CAD models characteristically present geometrical discontinuities. These geometrical discontinuities will have a great influence on the toolpath continuity inducing marks on the finished surface. These marks affect the functionality of the finished surfaces. Chaves-Jacob et al. [14] carried out a design of experiment to highlight the effect of the CAD surface discontinuities on the machined surface quality.

Currently a toolpath is commonly defined by a sequence of ordered points linked by linear trajectories. Certainly, a slope discontinuity is present at each toolpath point. To solve this problem, new interpolations are developed. For example, a sliding five-degree polynomial interpolation was proposed to smooth toolpath discontinuities and accordingly surface smoothness [14]. Other interpolations are developed like B-Spline interpolation. Such approaches are presented by Langeron et al. [15] and Lartigue et al. [16]. In this case, interpolation is integrated in an industrial CAM system. Furthermore, Yong et al. [17] used a curve interpolation to smooth slope discontinuities. Actually, the authors added a B-Spline corner to increase the feed rate.

On the other hand, the distance between two consecutive points of a toolpath may induce a reduction of the feed rate in high speed machining. Lavernhe et al. [18] presented a predictive model of kinematic behaviour for a 5-axis machine. In this study, the author explains that to avoid a reduction of the feed rate, a minimal distance between two consecutive points must be ensured. This distance depends on the CNC computer performance.

Figure 2 highlights the main problems of a toolpath computed on a meshed surface: The first problem is the large discontinuities in the slope of the generated toolpath segments. By nature, meshed surfaces present slope discontinuities between two facets. These discontinuities will evidently induce large slope discontinuities in the toolpath.

The second problem is the non-homogeneous density of points along the toolpath and the presence of overabundant points. The CAM system computes a toolpath to ensure an objective deviation between the nominal surface and the computed toolpath. Generally, this acceptable deviation is called chordal error. Figure 2 illustrates the toolpath of the contact points between the tool and the surface computed using an iso-planar strategy. To compute this toolpath, the CAM system begins computing the intersection line between a plane and the nominal surface. Next, the CAM system computes points on this intersection line; these 
points are the cutter contact points of the toolpath. The distance between these points is determined to ensure the chordal error. Finally, the CAM system computes the coordinates of the drive point. In the case of meshed surface machining, the intersection line is a succession of segments (each segment is the intersection of the plane and a triangular facet). Naturally, these segments will become the cutter contact toolpath with a chordal error equal to zero. In this case, the segment length is determined by the position of the plane relative to the triangle facet. Accordingly, the repartition of the point along the toolpath is not relevant and is minimal to its geometrical definition as illustrated in figure 2 .

This study proposes toolpath smoothing using a polynomial interpolation combined with a concatenation method. Proposed method may be employed with high end controllers commonly used on 5-axis CNC machines. The concatenation method smoothes sets of initial toolpath points by polynomial segments. This method increases the size of each polynomial segment and reduces the number of segments. This concatenation is carried out to ensure a specified tolerance. The aim of both proposed methods is to improve the quality of the machined surface. The proposed method carries out the smoothing of an unsmooth toolpath. Thereby, it is appropriated to solve the two mains problems of toolpath computed on meshed surfaces but it may also be used to improve the quality of any toolpath. The proposed method is useful and may improve the quality of the toolpath computed on unsmooth surfaces. The method may be used with any type of tool. Indeed, Vickers et al. [19] highlighted the importance of the type of milling tool on the machining efficiency in end milling. Furthermore, in the proposed method the gouging problem is handled during the initial computation of the toolpath. In fact, the deviations introduced by the toolpath smoothing are small and can be neglected in the gouging phenomenon. The combination of polynomial interpolation and concatenation methods permits machining meshed free-form surface without surface reconstruction.

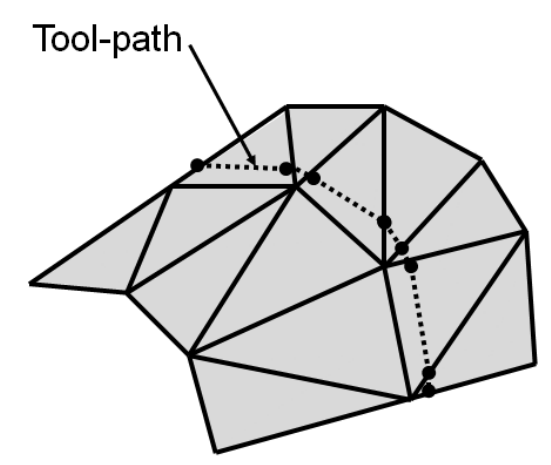

Fig.2 The main problems of the toolpath computed on meshed surfaces. 
This study is composed of three sections. The first section presents the polynomial interpolation method. This interpolation ensures continuity in slope and curvature over the whole toolpath. This method generates a smooth toolpath which may be read by common CNC machines. Moreover, this interpolation keeps the initial toolpath points. Subsequently, a concatenation method is developed to reduce the size of the CNC program and to improve the smoothing of the toolpath. This method automatically suppresses the toolpath points that are not necessary to obtain the required quality. Finally, the efficiency of both combined methods is tested by machining a biomedical meshed surface. The presented method is compared to solutions already implemented in actual CNC machines. This experimental machining test highlights the quality of the machined surface obtained by the proposed method.

\section{Proposed polynomial interpolation method}

In industry the most commonly used interpolation is the linear interpolation between two consecutive points of the toolpath. In addition, common CNC machine controllers are also able to perform polynomial and B-spline interpolations. Polynomial interpolation is proposed to smooth unsmooth toolpaths. Certainly, toolpaths computed on CAD models that include geometrical discontinuities (e.g. Meshed models) will not be smoothed. This type of toolpath induces marks on the machined surface that affect the functionality of the surface. The aim of the proposed method is to smooth the initial toolpath with a soft polynomial interpolation. Two classical mathematical formulations are available on CNC machine controllers: standard polynomial interpolation and Bspline fitting. Standard polynomial interpolation was however selected for his main advantage: the simplicity for an operator in front of the CNC machine to read the program. Indeed, Bspline formulation uses control points that do not belong to the real toolpath, what disturbs the reading.

The proposed method starts from a linear interpolation toolpath as computed by common CAM software. The aim of the method is then to associate a smooth polynomial curve between two successive points of the linear toolpath. The proposed interpolation is defined to improve the mathematical continuity of the toolpath. It ensures the continuity of the toolpath in both slope and curvature and consequently leads to smooth variations of the tool acceleration. In fact, the minimal polynomial degree required to ensure such conditions is three. Indeed, the polynomial must cross the start and end points of a given interpolation interval (e.g. $M_{i}$ and $M_{i+1}$ in Figure 3). At each toolpath point, the slope and curvature calculated from the left and right interpolation polynomials are also to be the same. These four constraints impose thus a minimum polynomial degree of three. However, with no additional constraint the slope and curvature of each toolpath point are then fixed by the coordinates of the start and end points of the left interpolation interval. This leads to spurious 
undulations. To overcome, this impediment the slopes and curvatures were imposed (with a robust method: parabolas) at each point of the toolpath. These conditions increase to six the number of constraints of each polynomial (i.e. fixed coordinates, slopes and curvatures of the start and end points of each interpolation interval), thus leading to a five degree formulation. The sliding five-degree polynomial toolpath is used to smooth the path between the successive points of the initial toolpath. Figure 3 presents the used nomenclature. Parabolas are used to control the slope and the curvature in the link between two consecutive five degree polynomial curves. These parabolas are a mathematical artefact. A Parabola is the simplest mathematical curve to compute the curvature at a given point. Furthermore, the computation of a parabola through three points is very robust. At point $M_{i}$ a parabola is computed to cross the three points $M_{i-1}, M_{i}$ and $M_{i+1}$. Eq. 1 gives the expression of the parabola. This procedure defines the slopes and curvatures to be imposed at the start and end points of the five-degree interpolation polynomial. Accordingly, the continuity in slope and curvature of the whole toolpath is ensured.

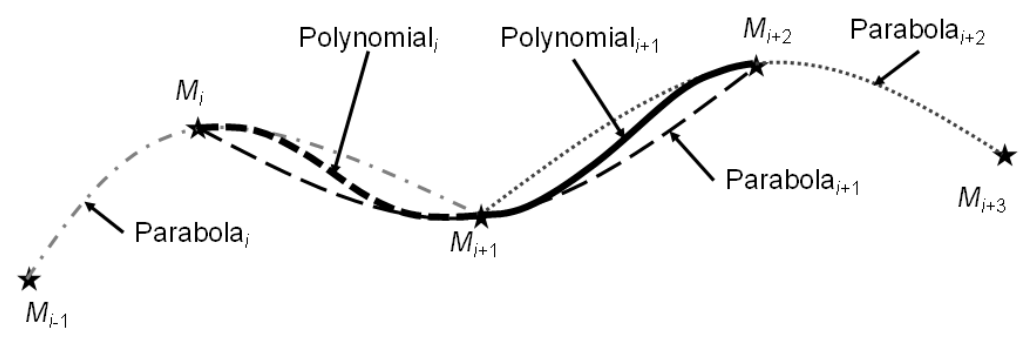

Fig.3 Interpolation on a segment

Subsequently, computations are presented for the $X$-axis only. The same treatment is however applied to $Y$ and $Z$ axes to obtain the toolpath.

$$
\operatorname{parabolạ}(u) \cdot X=d_{0}+d_{1} \cdot u+d_{2} \cdot u^{2}
$$

The value of the parameter $u$ at each point must be chosen to determine the coefficients $d_{0}, d_{1}$ and $d_{2}$ of Eq. 1 . The parameter $u$ is imposed to -1 at point $M_{i-1}, 0$ at point $M_{i}$ and 1 at point $M_{i+1}$. These choices produce the results presented in Eq. 2.

$$
\left\{\begin{array}{l}
x_{M i}=d_{0} \\
x_{M i-1}=d_{0}-d_{1}+d_{2} \\
x_{M i+1}=d_{0}+d_{1}+d_{2}
\end{array}\right.
$$


The coefficients of the parabola are determined solving Eq. 2. The results are presented in Eq. 3. This equation highlights that the coefficients of the parabola are only determined by the initial toolpath coordinates.

$$
\left\{\begin{array}{l}
d_{0}=x_{M i} \\
d_{1}=\frac{x_{M i+1}-x_{M i-1}}{2} \\
d_{2}=\frac{x_{M i+1}+x_{M i-1}}{2}-x_{M i}
\end{array}\right.
$$

Subsequently, the first and second derivatives of the parabola $i$ are computed at $M_{i}$. The results of this computation are presented in Eq. 4. These values are respectively written $T x_{i}$ and $C x_{i}$ and are associated with point $M_{i}$ to compute the polynomial interpolation.

$$
\left\{\begin{array}{l}
\frac{\mathrm{d}(\text { Parabola })}{\mathrm{d} u}(0)=T x_{i}=d_{1} \\
\frac{\mathrm{d}^{2}(\text { Parabola })}{\mathrm{d} u^{2}}(0)=C x_{i}=2 \cdot d_{2}
\end{array}\right.
$$

Eq. 5 presents the mathematical definition of a five-degree polynomial. One polynomial is defined between each $M_{i} M_{i+1}$ interval. For example, if the initial toolpath has 10 points this method defines 9 polynomials. Each polynomial is driven by the parameter named $p$. Subsequently, an arbitrary choice of the $p$ value at $M_{i}$ and $M_{i+1}$ is carried out, $p$ is selected to have the value $p=0$ at $M_{i}$ and $p=1$ at $M_{i+1}$. Accordingly, the polynomial curve is imposed to cross the points $M_{i}$ and $M_{i+1}$. Furthermore, the first and second derivatives of the polynomial toolpath at $M_{i}$ are imposed respectively equal to $T x_{i}$ and $C x_{i}$. Certainly, a five-degree polynomial curve between intervals $M_{i} M_{i+1}$ has 6 unknown parameters. On the other hand, this curve is submitted to 6 constraints:

- pass through $M_{i}$ and $M_{i+1}$;

- first derivatives at $M_{i}$ and $M_{i+1}$ are imposed, respectively equal to that of the parabola and parabola $i_{i+1}$;

- second derivatives at $M_{i}$ and $M_{i+1}$ are imposed respectively equal to that of the parabola and parabola $a_{i+1}$.

Eq. 6 synthesis all these constraints in a matrix form.

$$
\operatorname{Poly}_{\mathrm{i}}(p) \cdot X=a_{0}+a_{1} \cdot p+a_{2} \cdot p^{2}+a_{3} \cdot p^{3}+a_{4} \cdot p^{4}+a_{5} \cdot p^{5}
$$




$$
\left[\begin{array}{cccccc}
1 & 0 & 0 & 0 & 0 & 0 \\
1 & 1 & 1 & 1 & 1 & 1 \\
0 & 1 & 0 & 0 & 0 & 0 \\
0 & 1 & 2 & 3 & 4 & 5 \\
0 & 0 & 2 & 0 & 0 & 0 \\
0 & 0 & 2 & 6 & 12 & 20
\end{array}\right] \bullet\left[\begin{array}{c}
a_{0} \\
a_{1} \\
a_{2} \\
a_{3} \\
a_{4} \\
a_{5}
\end{array}\right]=\left[\begin{array}{c}
x_{M i} \\
x_{M i+1} \\
T x_{i} \\
T x_{i+1} \\
C x_{i} \\
C x_{i+1}
\end{array}\right]
$$

Eq. 6 is solved to determine the equation of the five degree polynomial toolpath between $M_{i}$ and $M_{i+1}$. The last presented computations are only conducted for the $X$ axis. Eq. 7 presents the mathematical form of the five degree polynomial for the three dimensions. The presented resolution of Eq. 6 in three dimensions enables the determination of all the polynomial coefficients of Eq. 7. This polynomial interpolation is computed for all $M_{i} M_{i+1}$ intervals built with the successive points of the initial toolpath.

$$
\operatorname{Poly}_{i}(p)=\left\{\begin{array}{l}
x(p)=a_{0}+a_{1} \cdot p+a_{2} \cdot p^{2}+a_{3} \cdot p^{3}+a_{4} \cdot p^{4}+a_{5} \cdot p^{5} \\
y(p)=b_{0}+b_{1} \cdot p+b_{2} \cdot p^{2}+b_{3} \cdot p^{3}+b_{4} \cdot p^{4}+b_{5} \cdot P^{5} \\
z(p)=c_{0}+c_{1} \cdot p+c_{2} \cdot p^{2}+c_{3} \cdot p^{3}+c_{4} \cdot p^{4}+c_{5} \cdot p^{5}
\end{array}\right\}
$$

The characteristic of this method is the use of parabolas to determine the slope and curvature in the junction between two polynomial interpolations. The use of parabolas avoids the apparition of oscillations and leads to a great stability of the computed toolpath. This type of toolpath ensures thus a high quality of the machined surface.

Subsequently, a CNC code is generated to be downloaded to the CNC machine. Most CNC machines are able to read polynomial interpolations. Eq.8 presents a line of CNC program built for a SIEMENS $840 \mathrm{D}$ controller. The coefficients $a_{0}, a_{1}, b_{0}, b_{1}, c_{0}$ and $c_{1}$ are not provided in the program. These coefficients are linked to the current and end coordinates of each interpolation interval. The CNC program lines are not too complicated to write and can be easily read by a CNC operator.

$\mathrm{POLY} X=\mathrm{PO}\left(X_{\mathrm{Mi+1}}, a_{2}, a_{3}, a_{4}, a_{5}\right) \mathrm{Y}=\mathrm{PO}\left(Y_{\mathrm{Mi}+1}, b_{2}, b_{3}, b_{4}, b_{5}\right) \mathrm{Z}=\mathrm{PO}\left(Z_{\mathrm{Mi}+1}, c_{2}, c_{3}, c_{4}, c_{5}\right) \mathrm{PL}=1$

\section{Concatenation method}

Section $\S 2$ proposed a method to compute a smooth polynomial interpolation between points of a toolpath. Certainly, this method defines a five-degree polynomial between the successive points of the initial toolpath. The aim of the concatenation method is to increase 
the length of each polynomial segment. The concatenation method is based on the skipping of unnecessary points. Points are skipped while a specified tolerance $\left(I T_{C}\right)$ is maintained between the skipped points and the polynomial toolpath. The concatenation method generally has four objectives:

- To reduce the CNC program file size to avoid memory overflow, this problem is typical of complicated workpieces.

- To reduce the number of links. This will increase the toolpath quality and accordingly reduce the acceleration and deceleration steps. Consequently, the feed-rate can be increased and will be more stable, reducing the machining time.

- To get a homogeneous density of points along the toolpath, thus contributing to improve the toolpath quality.

- To skip close points of the initial toolpath. These close points may create feed rate decelerations along the toolpath inducing machining marks on the surface. Naturally, these close points can be skipped without loss of geometrical quality.

The idea behind this method is to skip initial toolpath points to increase the size of the polynomial, respecting a specified tolerance $\left(I T_{C}\right)$. Figure 4 illustrates this idea.

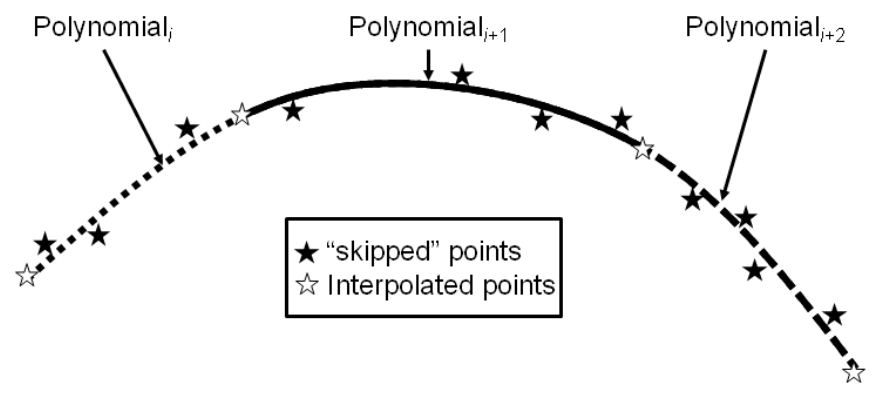

Fig. 4: concatenation method.

Figure 5 illustrates the main steps of the concatenation method. The algorithm presents the procedure to determine a polynomial toolpath between the points $M_{\mathrm{i}}$ and $M_{\mathrm{a}}$. This iterative algorithm successively skips points as long as the error between each skipped point and the polynomial toolpath remains less than a specified tolerance $I T_{\mathrm{C}}$. Eq. 9 defines the value of the distance $e_{i}$ between a toolpath point and the polynomial toolpath. Indeed, this distance is an approximation of the real error made on the workpiece. Authors like Huang et al. [20] propose to compute the real interferences caused by the chordal error. These computations need however to know the true shape of the machined surface. In the presented case only the toolpath is known to be smoothed. Furthermore, the specified tolerance $\left(I T_{C}\right)$ is selected small in comparison to the machining tolerance. 


$$
e_{i}=\operatorname{distance}\left[M_{i} ; \operatorname{Poly}_{i}(p)\right]
$$

The slope and curvature properties at point $M_{\mathrm{a}}$ are computed using a parabola, which crosses the points $M_{\mathrm{i}}, M_{\mathrm{a}}$ and $M_{2 a-i}$. The iterative algorithm stops when: the tolerance is exceeded or the index of the last point of the initial toolpath is reached. When the tolerance is exceeded the polynomial toolpath is computed for the correct value of index $a$. The method used to compute the five-degree polynomial toolpath was already presented in section $\S 2$.

The presented concatenation method ensures a smooth toolpath. Furthermore, this method provides a reduced number of segments to ensure a specified tolerance between the nominal points and the concatenate toolpath.

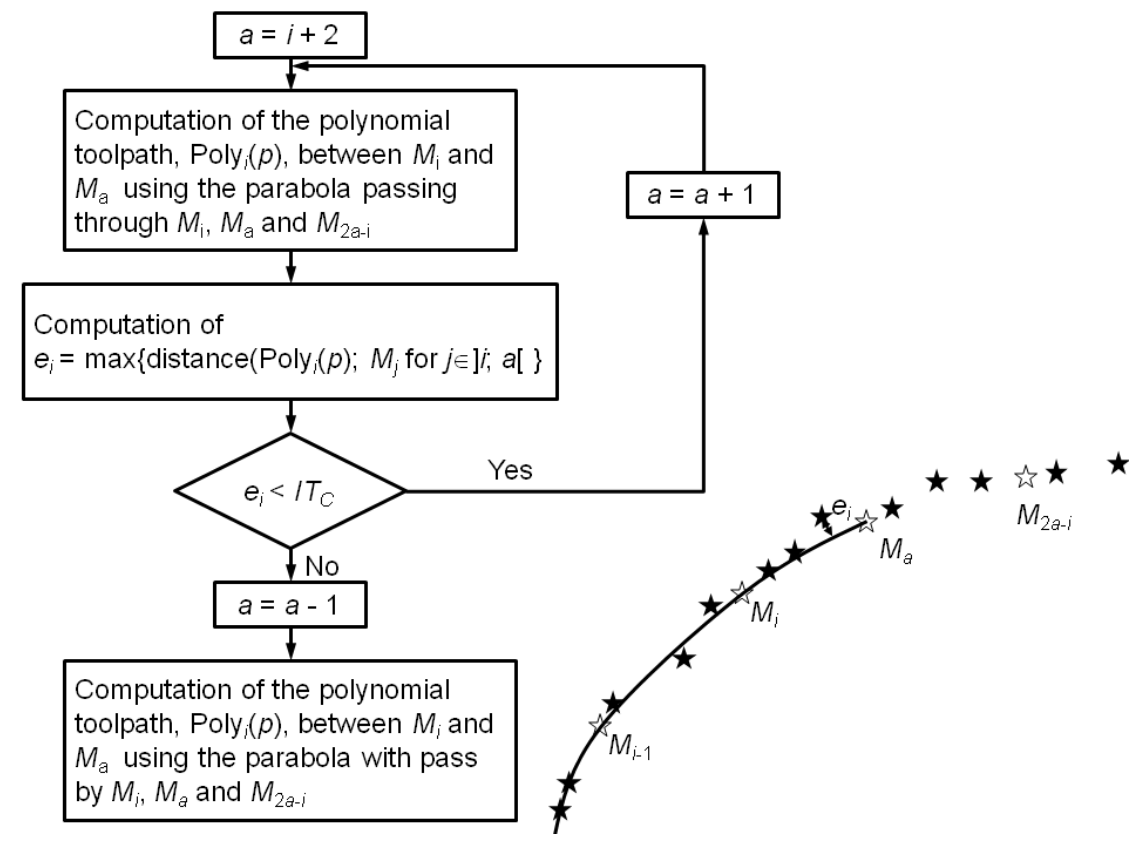

Fig.5: Flowchart of the concatenation method

\section{4) Application}

The Methods presented in sections $\S 2$ and $\S 3$ are used to machine a part of the tibial prosthesis plateau presented in Figure 6 . The area of the friction surface machined for the tests is presented in this figure. An STL model with a small facet size defines the surface used to compute the toolpath. The toolpath is computed with industrial CAM software. An iso-planar strategy with $0.16 \mathrm{~mm}$ of step-over is used for that purpose. This STL model induces a toolpath with a lot of very close points. The machining was carried out in an aluminium alloy with a ball-end-tool with a diameter of $10 \mathrm{~mm}$. The employed tool has four 
flutes. The spindle rotation speed was fixed to $10000 \mathrm{rpm}$ and the feed rate was set to $3000 \mathrm{~mm} / \mathrm{min}$ (feed per tooth of $0.075 \mathrm{~mm}$ ). Linear and polynomial toolpaths were employed and compared in this experiment. Three types of toolpaths were considered:

- The basic linear interpolation toolpath called G1 in table 1. This is the standard linear interpolation available in the CNC machine without using any enhanced interpolation option. This type of toolpath is not used to mill workpieces because it creates lot of vibration during machine displacement.

- The linear interpolation toolpath, named G1 + CNC options in table 1. This interpolation is the current method used in industry. It is a linear interpolation using CNC options to smooth free-form surfaces. In this case: G642 and COMPCAD commands of a SIEMENS 840D NC controller were used.

- The polynomial interpolation combined with a concatenation with $I T_{\mathrm{C}}$ tolerance (methods presented in sections $\S 2$ and $\S 3$ ). This interpolation is defined by the value of $I T_{\mathrm{C}}$ in table 1 . In table 1 , the strategy with POLY and $I T_{\mathrm{C}}$ equal to zero corresponds to a polynomial interpolation without concatenation.

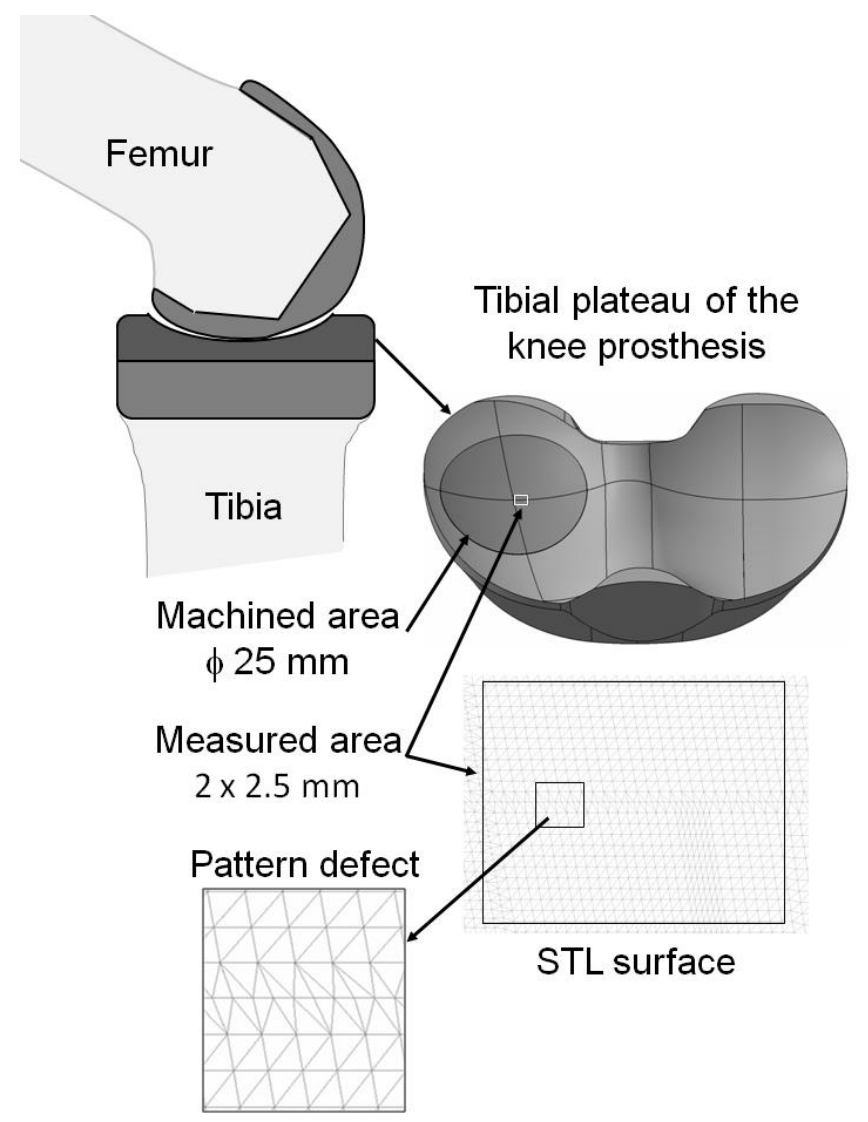

Fig.6: definition of the machined and measured areas of the tibial plateau prosthesis 
Careful Roughness measurements were carried out using an optical chromatic confocal sensing Coordinate Measuring Machine (CMM). The resolution of the CMM sensor is about $10 \mathrm{~nm}$. The Measurements were done on a $2 \times 2.5 \mathrm{~mm}$ patch with a step of $4 \mu \mathrm{m}$ in both directions. Figure 6 presents the position of the measured area. To highlight the differences between the tested toolpaths, this area was selected along a local STL pattern defect. The form of the measured surface was filtered with a three degree polynomial surface. Then residuals of the best fit were used to compute the two roughness parameters, $\mathrm{Sa}$ (arithmetic average) and $S z$ (maximum height) presented in table 1.

Furthermore, table 1 presents:

- The number of toolpath points: this value expresses the number of non skipped points.

- The average length between two successive points of the toolpath. This parameter highlights that the initial toolpath (G1 in table 1$)$ has very close points $(0.1561 \mathrm{~mm})$.

- The machining time is measured directly on the CNC machine for all toolpath strategies. Some experiments are conducted displacing the tool without workpiece. Since all the strategies have the same toolpath length, the execution time of such experiment is then used to characterize the real feed rate.

- The roughness parameters: The tests without workpiece have grey cells in these two columns.

Table 1 highlights the efficiency of the classical smoothing technique already incorporated in CNC options. Certainly, the machining time between the standard linear interpolation and the smoothed linear interpolation using CNC options is divided by more than five. The reduction of the machine tool feed rate may explain this phenomenon. Without CNC options, the machine must slow to exactly follow the specified points and undergoes all the geometrical discontinuities of the toolpath. In the STL file, the short distance between two successive points highlights this phenomenon.

Furthermore, this table shows that the polynomial interpolation without concatenation $\left(I T_{\mathrm{C}}\right.$ equal to zero) is not appropriated for small segments. Certainly, the machining time and the surface roughness are significantly increased compared to the CNC options toolpath.

\begin{tabular}{|c|c|c|c|c|c|c|}
\hline $\begin{array}{c}\text { Type of } \\
\text { interpolation }\end{array}$ & $I T_{C}(\mathrm{~mm})$ & $\begin{array}{c}\text { Point } \\
\text { number }\end{array}$ & $\begin{array}{c}\text { Average } \\
\text { length }(\mathrm{mm})\end{array}$ & $\begin{array}{c}\text { Machining } \\
\text { time }(\mathrm{s})\end{array}$ & Sa $(\mu \mathrm{m})$ & $S z(\mu \mathrm{m})$ \\
\hline G1 & N/A & 15959 & 0.1561 & 848 & & \\
\hline $\begin{array}{c}\text { G1+CNC } \\
\text { options }\end{array}$ & N/A & 15959 & 0.1561 & 165 & 0.8 & 7.2 \\
\hline Poly & 0 & 15959 & 0.1561 & 231 & 1.23 & 10.6 \\
\hline Poly & 0.001 & 8440 & 0.2159 & 193 & & \\
\hline
\end{tabular}




\begin{tabular}{|l|l|l|l|l|l|l|}
\hline Poly & 0.002 & 7017 & 0.355 & 178 & & \\
\hline Poly & 0.003 & 6620 & 0.3763 & 176 & 0.762 & 6.55 \\
\hline Poly & 0.004 & 6214 & 0.4009 & 182 & & \\
\hline Poly & 0.006 & 6389 & 0.3899 & 185 & 0.674 & 8.05 \\
\hline Poly & 0.008 & 5868 & 0.4245 & 180 & & \\
\hline Poly & 0.01 & 5104 & 0.4881 & 172 & 0.704 & 5.80 \\
\hline Poly & 0.02 & 4414 & 0.5644 & 174 & & \\
\hline Poly & 0.04 & 3413 & 0.73 & 169 & 0.806 & 6.43 \\
\hline Poly & 0.06 & 2898 & 0.8597 & 168 & & \\
\hline
\end{tabular}

Table.1: Influence of the concatenation tolerance on the machining time and surface roughness.

Figure 7 was created to facilitate reading of table 1. This figure superimposes two graphs to highlight the correlations between two phenomena:

- The machining time versus the concatenation tolerance. This graph is composed of two curves: a solid line for the polynomial interpolation and a dotted line for the linear interpolation with CNC options (this curve is constant versus $I T_{\mathrm{C}}$ ).

- The roughness maximum height versus the concatenation tolerance. This plot is also composed of two curves.

Table 1 and figure 7 show that increasing the concatenation tolerance reduces the machining time of polynomial interpolation. Furthermore, the polynomial interpolation presents an optimal value: in this case $I T_{C}=0.01 \mathrm{~mm}$. At this configuration, the roughness is the lowest and the machining time is close to the one obtained with the smoothing technique already implemented in the CNC options. Furthermore, this figure highlights that the studied method almost always improves the surface quality without significantly increasing the machining time. 


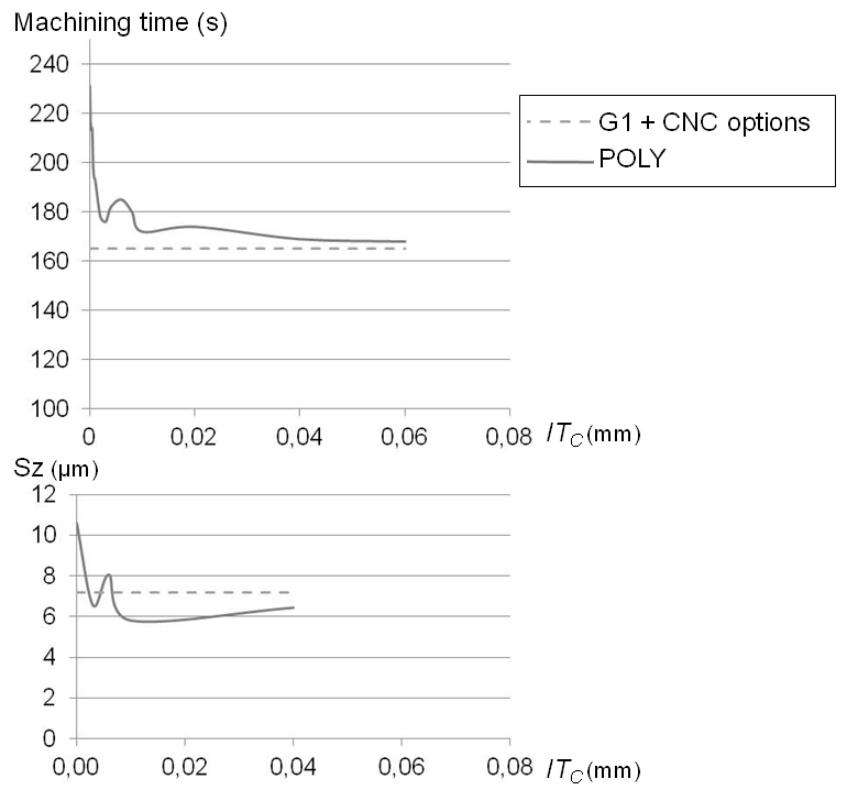

Fig 7: Evolution of the machining time and surface roughness versus the concatenation tolerance.

Figure 8 presents the measured surface micro-geometry (after form filtering) for the six machined surfaces. This roughness morphology is typical of iso-planar strategies on convex surfaces. Indeed, with such strategy, the tool axes stay in a plane during one pass but the cutter contact realises a trajectory that is curved in direction of the lowest point of the convex surface. Figure 7 highlights that in this case the optimal concatenation tolerance is $0.01 \mathrm{~mm}$. For this concatenation tolerance a smooth surface was obtained and the roughness parameters $S a$ and $S z$ were significantly decreased, respectively of $12 \%$ and $19 \%$.
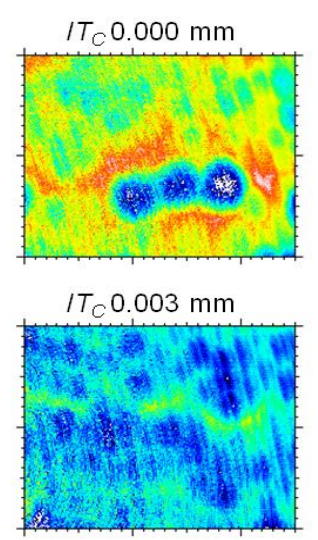

$$
1 T_{C} 0.006 \mathrm{~mm}
$$

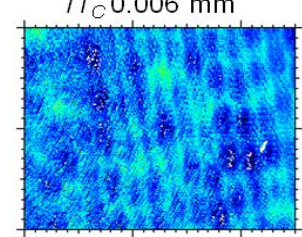

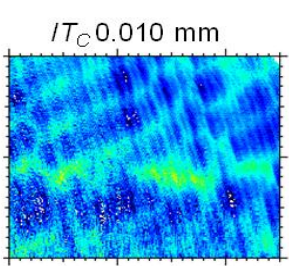

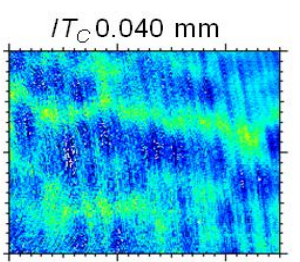

$\mathrm{G} 1+\mathrm{CNC}$ options

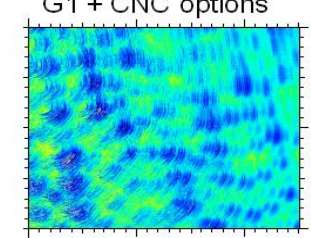

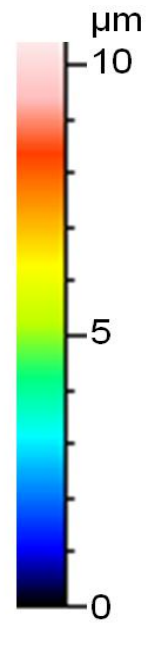

$\mu \mathrm{m}$

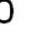

Fig 8: Micro-geometry of the measured surface. 
The experimental tests illustrate the usefulness of the methods presented in sections §2 and §3. They highlight that the basic linear interpolation cannot be used to machine free-form surfaces defined by a meshed surface. Furthermore, in this case, the experimental test shows that the CNC options provide good machining time but the surface quality is not optimal. On the other hand, the test carried out with the proposed method (enhanced polynomial interpolation \& concatenation) increases the surface quality with a similar machining time. Furthermore, an optimal tolerance value is found to carry out the best machining performance. Accordingly, the presented methods are efficient for machining meshed surfaces.

\section{Conclusion}

This study presents a method to efficiently smooth toolpaths computed on meshed surfaces. Naturally, such toolpaths present large slope discontinuities and a nonhomogeneous points repartition along the trajectory. A method was therefore proposed to overcome this impediment. The developed method is based on an enhanced five-degree polynomial interpolation. This interpolation is computed to ensure the continuity in slope and curvature of the computed toolpath. The geometrical continuity of the toolpath ensures smooth variations of the acceleration along the toolpath and consequently improves the quality of the machined surface. The proposed method may be employed with high end controllers commonly used on 5-axis CNC machines. This interpolation is coupled with a concatenation method. The aim of this method is to reduce the size of the CNC program and to remove overabundant points. This method is based on point skipping in order to increase the length of the polynomial segments. It however limits the maximum deviation between the original trajectory and the concatenated toolpath. To validate the approach, the usefulness of these two methods was tested for the machining of tibial plateau prostheses. In these tests, the CAD model of the workpiece is defined by a meshed model ( $\left.{ }^{*} . S T L\right)$. The experiments highlight that the proposed method presents an optimal value of concatenation tolerance. The results show that the proposed method provides the same machining time as the classical smoothing technique already incorporated in CNC options and currently used in industry, but better surface roughness.

\section{References:}

[1] Lim SP, Haroun H (2012) Surface reconstruction techniques: a review. Artificial Intelligence Review. doi: 10.1007/s10462-012-9329-z 
[2] Park SC, Chung YC (2000) Tool-path generation from measured data. Computer-Aided Design 35: 467-475. doi: 10.1016/S0010-4485(02)00070-2

[3] Zhang Y, Ge L (2011) Adaptive tool-path generation on point-sampled surfaces.

Precision. Engineering-Journal of the International Societies 35: 591-601. doi:

10.1016/j.precisioneng.2011.04.002

[4] Feng HY, Teng ZJ (2005) Iso-planar piecewise linear NC tool path generation from discrete measured data points. Computer design 37: 55-64. doi :10.1016/j.cad.2004.04.001

[5] Chui K L, Chiu W K, Yu K M (2008) Direct 5-axis tool-path generation from point cloud input using 3D biarc fitting. Robotic and Computer Integration 24: 270-286. doi:

10.1016/j.rcim.2006.11.004

[6] Sun YW, Guo DM, Jia ZY, et al.(2006) Iso-parametric tool path generation from triangular meshes for free-form surface machining. International Journal of Advenced manufacturing 28: 721-726. doi: 10.1007/s00170-004-2437-4

[7] Park, SC (2004) Sculptured surface machining using triangular mesh slicing. ComputerAided Desgn. 36: 279-288. doi: 10.1016/S0010-4485(03)00114-3

[8] Jun CS, Kim DS, Park SC (2002) A new curve-based approach to polyhedral machining. Computer-Aided Design 34: 379-389. doi: 10.1016/S0010-4485(01)00110-5

[9] Kim SJ, Yang MY (2006) A CL surface deformation approach for constant scallop height tool path generation from triangular mesh. The International Journal of Advanced Manufacturing Technology 28: 314-320. doi: 10.1007/s00170-004-2372-4

[10 ] Lauwers B, Kiswanto G, Kruth JP (2003) Development of a Five-axis Milling Tool Path Generation Algorithm based on Faceted Models. CIRP Annals - Manufacturing Technology 52/1: 85-88. doi: 10.1016/S0007-8506(07)60537-X

[11] Lu J, Cheatham R, Jensen CG, Chen Y, Bowman B (2008) A three-dimensional configuration-space method for 5 -axis tessellated surface machining. International Journal of Computer Integrated Manufacturing 21/5: 550-568. doi: 10.1080/09511920701263313 [12] Kiswanto G, Lauwers B, Kruth JP (2007) Gouging elimination through tool lifting in tool path generation for five-axis milling based on faceted models. The International Journal of Advanced Manufacturing Technology 32/3-4: 293-309. doi: 10.1007/s00170-005-0338-9

[13] Lasemi A, Xue D, Gu P (2010) Recent development in NC machining of freeform surfaces: A stat-of-the-art review. Comput-Aided Des 42: 641-651. doi:

10.1016/j.cad.2010.04.002

[14] Chaves-Jacob J, Linares JM, Sprauel JM (2011) Increasing of surface quality in friction free-form surfaces of knee prosthesis. CIRP Annals - Manufacturing Technology. 60: 531534. doi: 10.1016/j.cirp.2011.03.059 
[15] Langeron JM, Duc E, Lartigue C, Bourdet P (2004) A new format for 5-axis tool path computation, using Bspline curves. Comput-Aided Des 36: 1219-1229. doi:

10.1016/j.cad.2003.12.002

[16] Lartigue C, Tournier C, Ritou M, Dumur D (2004) High-Performance NC for HSM by means of Polynomial Trajectories. CIRP Annals - Manu Tech 53: 317-320. doi:

10.1016/S0007-8506(07)60706-9

[17] Yong TH, Narayanaswami R (2003) A parametric interpolator with confined chord errors, acceleration and deceleration for NC machining. Computer-Aided Design 35: 1249-1259. doi: 10.1016/S0010-4485(03)00043-5

[18] Lavernhe S, Tournier C, Lartigue C (2008) Kinematical performance prediction in multiaxis machining for process planning optimization. International Journal of Manufacturing Technologie 37 : 534-544. doi: 10.1007/s00170-007-1001-4

[19] Vickers GW, Quan KW (1989) Ball-Mills Versus End-Mills for Curved Surface Machining. Journal of engineering for industry-transactions of the asme 111/1: 22-26.

doi:10.1115/1.3188728

[20] Huang YC, Oliver JH, Non-constant parameter nc tool path generation on sculptured surfaces. The international journal of advanced manufacturing technology 9/5: 281-290. doi: 10.1007/BF01781282 